DR MARTIN HOENIGL (Orcid ID : 0000-0002-1653-2824)

Article type : Original Article

\title{
Clinical Evaluation of the Newly Formatted Lateral- Flow Device for Invasive Pulmonary Aspergillosis
}

Running head: Newly formatted LFD for invasive aspergillosis

Martin Hoenigl 1,2,3,4\#, Susanne Eigl ', Sven Heldt ', Wiebke Duettmann ', Christopher Thornton ${ }^{5}$, Juergen Prattes ${ }^{2,4 \#}$

${ }^{1}$ Division of Pulmonology, Medical University of Graz, Graz, Austria

${ }^{2}$ Section of Infectious Diseases and Tropical Medicine, Medical University of Graz, Graz, Austria

${ }^{3}$ Division of Infectious Diseases, Department of Medicine, University of CaliforniaSan Diego, San Diego, USA

${ }^{4}$ CBmed - Center for Biomarker Research in Medicine, Graz, Austria

${ }^{5}$ Biosciences, University of Exeter, Exeter, United Kingdom

This article has been accepted for publication and undergone full peer review but has not been through the copyediting, typesetting, pagination and proofreading process, which may lead to differences between this version and the Version of Record. Please cite this article as doi: 10.1111/myc. 12704

This article is protected by copyright. All rights reserved. 
\# Corresponding authors:

Martin Hoenigl, M.D., Section of Infectious Diseases and Tropical Medicine AND

Division of Pulmonology, Department of Internal Medicine,

Medical University of Graz, A- 8036 Graz, Austria.

Phone: +43 31638581319 Fax: +43 31638514622

E-mail: martin.hoenigl@medunigraz.at

\section{AND}

Juergen Prattes, M.D., Section of Infectious Diseases and Tropical Medicine,

Department of Internal Medicine,

Medical University of Graz, A- 8036 Graz, Austria.

Phone: +43 31638530046 Fax: +43 31638514622

E-mail: juergen.prattes@medunigraz.at

Key words: Haematological malignancy, bronchoalveolar lavage, Galactomannan, ICU, point-of-care. 


\section{Abstract}

The study evaluated the newly formatted Aspergillus-specific lateral-flow-device (LFD), and compared its performance to the original prototype "old" LFD test using BALF samples from 28 patients (14 patients with probable/proven invasive pulmonary aspergillosis [IPA] and 14 patients with no evidence for IPA). A total of $10 / 14(71 \%)$ of BALF samples from patients with probable/proven IPA resulted positive with the new LFD, including $8 / 9$ with true-positive and 2/5 with false-negative results with the old LFD. All 14 samples from patients without IPA resulted negative with the new LFD; specificity of the new LFD was significantly improved compared to the old LFD.

\section{Introduction}

Diagnosis of invasive pulmonary aspergillosis (IPA) during the early stages of disease enables targeted antifungal treatment and has the potential to significantly improve patient survival [1]. The Aspergillus-specific lateral-flow device (LFD) is an immuno-chromatographic assay that detects an extracellular glycoprotein antigen secreted during active growth of the pathogen [2]. The ease-of-use of the assay, requiring no pre-treatment of bronchoalveolar lavage fluid (BALF), allows point-ofcare testing, with results available within 15 minutes. To date, a prototype version of the test has been evaluated with more than 650 BALF samples across a number of studies, with an overall sensitivity of $73 \%$, specificity of $90 \%$, a positive predictive value (PPV) of $61 \%$, and negative predictive value (NPV) of $94 \%$ for probable/proven IPA versus no IPA [3-9]. As with other diagnostic tests for IPA such as the galactomannan [GM] ELISA, LFD sensitivity is reduced by mould-active antifungal 
drugs [10]. Despite this, it's accuracy in detecting IPA in hematological malignancy patients with probable/proven disease has been demonstrated [3,6,11], with a specificity of $91 \%$ and sensitivity of $67 \%$, and with additional discriminatory power for possible IPA, with $31 \%$ of cases negative by GM ELISA being positive by LFD [3].

Following extensive appraisal of the prototype LFD, the test has now been formatted for large-scale manufacture and CE marking as an in vitro diagnostic (IVD) device. The objective of this study was to clinically evaluate the newly formatted (hereafter referred to as new) LFD for the first time, and to compare its performance against the prototype (hereafter referred to as old) LFD using BALF samples from hematological malignancy and intensive care unit (ICU) patients.

\section{Methods:}

This study was conducted at the Medical University of Graz, Austria in June and July 2017. We tested a convenience series of 28 samples from adult patients, which were prospectively collected and tested with the old LFD (and also for GM) between November 2012 and September $2016[6,7,12,13]$ and subsequently stored at $-70^{\circ} \mathrm{C}$. The samples were selected based on underlying disease (principally hematological malignancy, secondarily ICU patients), IPA status according to 2008 EORTC/MSG criteria [14], and test result with the old LFD. All 28 samples were selected before the first sample was tested with the new LFD. Of the 14 BALF samples from patients with probable/proven IPA, 9 were positive with the old LFD (classified here as "true positives"), and 5 were negative with the old LFD (classified here as "false negatives"). Of the 14 BALF samples from patients without evidence for IPA, 9 were 
negative with the old LFD (classified here as 'true negatives'), and 5 patients were positive with the old LFD (classified here as 'false positives').

Testing with the new LFD (OLM Diagnostics, Newcastle upon Tyne, United Kingdom) was performed in our Microbiology Laboratory. Stored BALF samples where thawed, vortexed, and immediately tested without pre-treatment by applying $100 \mu \mathrm{L}$ of BALF to the test, with results read 15 minutes later, as described previously [7]. The interpreters of the LFD test results were blinded to IPA status and results of the old LFD, ensuring an unbiased interpretation of the test line results (Figure 1).

The study protocol was approved by the local ethics committee, Medical University Graz, Austria (EC-number 25-221 ex 12/13), and reported to the Austrian Agency for Health and Food Safety (Protocol number INS-621000-0478). Results of this study are reported according to the STARD statement.

Performance of the new LFD (including 95\% confidence intervals [CI]) was compared with that of the old LFD for differentiation between probable/proven IPA and no evidence for IPA using descriptive analysis and Fishers exact test. A twosided $p$-value of $<0.05$ was considered statistically significant.

\section{Results:}

A total of 28 patients (20 females, 8 males, median age 60 years, 23 underlying hematological disease, 5 ICU patients) were included in this analysis, of which 14 fulfilled the criteria for either proven $(n=3)$, or probable IPA $(n=11)$, and 14 patients had no evidence for IPA. $17 / 28(61 \%)$ of patients overall, and 7/14 (50\%) of patients with probable/proven IPA, were receiving mold-active antifungal prophylaxis/therapy at the time of BALF sampling. 
Characteristics and diagnostic test results for 14 patients with probable/proven IPA and those 5 with false positive old LFD results are depicted in Table 1 (details for those 9 patients without evidence for IPA and true negative results with the old LFD are given in the footnote of the Table). While the new LFD yielded results similar to those of the old LFD in 17/28 samples, overall result (i.e., positive versus negative) differed in 8/28 samples. In 3/28 BALF samples the results were consistently positive, but intensity of the test line differed.

A total of $10 / 14$ of BALF samples from cases with probable/proven IPA resulted positive with the new LFD (sensitivity 71\%; 95\%Cl 42-92\%), including 8/9 "true positives" and 2/5 "false negatives" with the old LFD. All 14 samples from cases with no evidence for IPA resulted negative with the new LFD (specificity $100 \% ; 95 \% \mathrm{CI}$ $77-100 \%)$. The specificity of the new LFD was significantly higher compared to the old LFD ( $p=0.04)$.

\section{Discussion:}

The Aspergillus-specific LFD test has now been formatted for large-scale manufacture and CE marking as an IVD for point-of-care diagnosis of IPA. This is the first study to clinically evaluate the newly formatted LFD and to compare its clinical performance with the old prototype test. We found that the new LFD when used with BALF samples was equally sensitive but more specific than the old LFD. The clinical sensitivity of $71 \%$ and specificity of $100 \%$ found in this analysis is remarkable considering the BALF selection process, which comprised samples that gave false negative or false positive results with the old LFD. In other words, the performance of the old LFD with the BALF samples selected for this evaluation (64\% 
sensitivity and $64 \%$ specificity), was inferior to the $73 \%$ sensitivity and $90 \%$ specificity published for the old BALF LFD over various patient cohorts, and also lower than the published performances of the old LFD in hematological malignancy patients (sensitivity $67 \%$, specificity $91 \%$; total $n=193$ samples), and ICU patients (sensitivity $79 \%$, specificity $85 \%$; total $n=239$ samples) [3].

The study has limitations of small sample size and sample pre-selection, and so has not determined the clinical performance of the new test in a prospectively tested cohort. Given that the old LFD was evaluated in a number of prospective multicenter studies, the direct comparison to results obtained with the old LFD may be the most valuable aspect of this work, but future larger prospective cohort studies are needed to confirm our findings. While BALF samples were tested with the new LFD months to years after sample collection and testing with the old LFD, it has been shown before that the LFD signal remains stable after several years of sample storage at $70^{\circ} \mathrm{C}[15]$

In conclusion, the newly formatted LFD may contribute to timely clinical decision making regarding initiation and choice of antifungal treatment. Taking into account test performance as well as its potential for point-of-care diagnosis, the new LFD represents a valuable addition to the currently available diagnostic arsenal for IPA. Considering the importance of rapid diagnosis and targeted treatment as major predictors of survival in patients with IPA, the potential clinical benefits of this new test become evident. 


\section{Conflicts of interest}

Martin Hoenigl has received a research grant for an investigator-initiated study from

Gilead, and speaker's honoraria from MSD, Gilead, and Basilea. Juergen Prattes has received consulting fees from Gilead. All other authors no conflict.

\section{Funding}

This work was supported by funds of the Oesterreichische Nationalbank (Anniversary Fund, project number 15346) and the Gilead Investigator Initiated Study IN-AT-131-1939. This work has also partly been carried out with the K1 COMET Competence Center CBmed, which is funded by the Federal Ministry of Transport, Innovation and Technology (BMVIT); the Federal Ministry of Science, Research and Economy (BMWFW); Land Steiermark (Department 12, Business and Innovation); the Styrian Business Promotion Agency (SFG); and the Vienna Business Agency. The COMET program is executed by the FFG (The Austrian Research Promotion Agency, project number 844609). LFD tests used in this study were provided by OLM Diagnostics. The funders had no role in study design, data collection, analysis, interpretation, decision to publish, in the writing of the manuscript, and in the decision to submit the manuscript for publication.

\section{References}

1. Cornely OA, Lass-Florl C, Lagrou K, Arsic-Arsenijevic V, Hoenigl M. Improving outcome of fungal diseases - Guiding experts and patients towards excellence. Mycoses 2017; 60: 420-425. 
2. Thornton CR. Development of an immunochromatographic lateral-flow device for rapid serodiagnosis of invasive aspergillosis. Clin Vaccine Immunol 2008; 15: 10951105.

3. Heldt S, Hoenigl M. Lateral Flow Assays for the Diagnosis of Invasive Aspergillosis: Current Status. Curr Fungal Infect Rep 2017; 11: 45-51.

4. Eigl S, Prattes J, Lackner M, et al. Multicenter evaluation of a lateral-flow device test for diagnosing invasive pulmonary aspergillosis in ICU patients. Crit Care 2015; 19: 178.

5. Willinger B, Lackner M, Lass-Florl C, et al. Bronchoalveolar lavage lateral-flow device test for invasive pulmonary aspergillosis in solid organ transplant patients: a semiprospective multicenter study. Transplantation 2014; 98: 898-902.

6. Prattes J, Lackner M, Eigl S, et al. Diagnostic accuracy of the Aspergillus-specific bronchoalveolar lavage lateral-flow assay in haematological malignancy patients. Mycoses 2015; 58: 461-469.

7. Prattes J, Flick H, Pruller F, et al. Novel tests for diagnosis of invasive aspergillosis in patients with underlying respiratory diseases. Am J Respir Crit Care Med 2014; 190: 922-929.

8. Hoenigl M, Prattes J, Spiess B, et al. Performance of galactomannan, beta-dglucan, Aspergillus lateral-flow device, conventional culture, and PCR tests with bronchoalveolar lavage fluid for diagnosis of invasive pulmonary aspergillosis. J Clin Microbiol 2014; 52: 2039-2045.

9. Miceli MH, Goggins MI, Chander P, et al. Performance of lateral flow device and galactomannan for the detection of Aspergillus species in bronchoalveolar fluid of patients at risk for invasive pulmonary aspergillosis. Mycoses 2015; 58: 368-374.

10. Eigl S, Prattes J, Reinwald M, et al. Influence of mould-active antifungal treatment on the performance of the Aspergillus-specific bronchoalveolar lavage fluid lateral-flow device test. Int J Antimicrob Agents 2015; 46: 401-405.

11. Hoenigl M, Koidl C, Duettmann W, et al. Bronchoalveolar lavage lateral-flow device test for invasive pulmonary aspergillosis diagnosis in haematological malignancy and solid organ transplant patients. J Infect 2012; 65: 588-591.

12. Eigl S, Hoenigl M, Spiess B, et al. Galactomannan testing and Aspergillus PCR in same-day bronchoalveolar lavage and blood samples for diagnosis of invasive aspergillosis. Med Mycol 2017; 55: 528-534.

13. Orasch T, Prattes J, Faserl K, et al. Bronchoalveolar lavage triacetylfusarinine $\mathrm{C}$ (TAFC) determination for diagnosis of invasive pulmonary aspergillosis in patients with hematological malignancies. J Infect 2017; May 31. Epub ahead of print.

14. De Pauw B, Walsh TJ, Donnelly JP, et al. Revised definitions of invasive fungal disease from the European Organization for Research and Treatment of

This article is protected by copyright. All rights reserved. 
Cancer/Invasive Fungal Infections Cooperative Group and the National Institute of Allergy and Infectious Diseases Mycoses Study Group (EORTC/MSG) Consensus Group. Clin Infect Dis 2008; 46: 1813-1821.

15. Prattes J, Koidl C, Eigl S, Krause R, Hoenigl M. Bronchoalveolar lavage fluid sample pretreatment with Sputasol((R)) significantly reduces galactomannan levels. $J$ Infect 2015; 70: 541-543. 
TABLE 1 Characteristics and Test Results (including those of the New LFD) in Patients with True Positive, False Negative, and False Positive Results with the Old LFD for Differentiation of Probable/Proven Invasive Pulmonary Aspergillosis versus No Evidence of IPA. Patients with true negative results $(n=9)$ are not displayed.*

\begin{tabular}{|c|c|c|c|c|c|c|c|c|c|}
\hline $\begin{array}{l}\text { Group } \\
\text { according } \\
\text { to old LFD } \\
\text { results and } \\
\text { IPA Status }\end{array}$ & $\begin{array}{l}\text { Pat } \\
\text { ient }\end{array}$ & $\begin{array}{l}\text { Primary underlying } \\
\text { disease }\end{array}$ & $\begin{array}{c}\text { Classifica } \\
\text { tion of } \\
\text { IPA } \S\end{array}$ & $\begin{array}{c}\text { AF } \\
\text { therapy/proph } \\
\text { ylaxis at the } \\
\text { time of } \\
\text { bronchoscopy }\end{array}$ & $\begin{array}{l}\text { BALF GM } \\
\text { ODI }\end{array}$ & $\begin{array}{l}\text { BALF culture } \\
\text { for moulds }\end{array}$ & $\begin{array}{c}\text { BALF } \\
\text { Aspergillus } \\
\text { specific } \\
\text { PCR results }\end{array}$ & Old LFD & New LFD \\
\hline \multirow{9}{*}{$\begin{array}{c}\text { TRUE } \\
\text { POSITIVES }\end{array}$} & 1 & Plasma cell leukemia & probable & no & 4.85 & neg & pos & +++ & +++ \\
\hline & 2 & $\begin{array}{l}\text { Acute myeloid } \\
\text { leukemia }\end{array}$ & probable & yes & 4.99 & neg & pos & + & ++ \\
\hline & 3 & $\begin{array}{l}\text { Acute myeloid } \\
\text { leukemia }\end{array}$ & probable & yes & 2.23 & neg & pos & + & + \\
\hline & 4 & Multiple myeloma & probable & no & 1.88 & neg & neg & ++ & + \\
\hline & 5 & $\begin{array}{l}\text { Chronic lymphoid } \\
\text { leukemia }\end{array}$ & probable & yes & 19.05 & neg & n.a. & ++ & - \\
\hline & 6 & ICU COPD & probable & no & 1.06 & neg & n.a. & ++ & + \\
\hline & 7 & ICU AIDS & proven & no & 17.0 & pos & pos & +++ & +++ \\
\hline & 8 & $\begin{array}{l}\text { ICU septic } \\
\text { pneumonia }\end{array}$ & probable & no & 5.59 & neg & pos & + & + \\
\hline & 9 & $\begin{array}{l}\text { ICU septic } \\
\text { pneumonia }\end{array}$ & probable & no & 4.62 & neg & neg & + & + \\
\hline \multirow{3}{*}{$\begin{array}{c}\text { FALSE } \\
\text { NEGATIVE } \\
\text { S }\end{array}$} & 10 & $\begin{array}{l}\text { Acute myeloid } \\
\text { leukemia }\end{array}$ & proven & yes & 0.37 & neg & neg & - & - \\
\hline & 11 & $\begin{array}{l}\text { Acute lymphoid } \\
\text { leukemia }\end{array}$ & probable & yes & 2.01 & neg & neg & - & + \\
\hline & 12 & $\begin{array}{l}\text { Acute myeloid } \\
\text { leukemia }\end{array}$ & probable & yes & 1.59 & neg & neg & - & + \\
\hline
\end{tabular}

This article is protected by copyright. All rights reserved. 


\begin{tabular}{|c|c|c|c|c|c|c|c|c|c|}
\hline & 13 & $\begin{array}{l}\text { Non-Hodgkin } \\
\text { lymphoma }\end{array}$ & probable & yes & 1.85 & neg & neg & - & - \\
\hline & 14 & $\begin{array}{l}\text { ICU septic } \\
\text { pneumonia }\end{array}$ & proven & no & 9.00 & pos & pos & - & - \\
\hline \multirow{5}{*}{$\begin{array}{c}\text { FALSE } \\
\text { POSITIVES }\end{array}$} & 15 & $\begin{array}{l}\text { Acute myeloid } \\
\text { leukemia }\end{array}$ & $\begin{array}{l}\text { no } \\
\text { evidence }\end{array}$ & yes & 0.09 & neg & neg & + & - \\
\hline & 16 & $\begin{array}{l}\text { Non-Hodgkin } \\
\text { lymphoma }\end{array}$ & $\begin{array}{l}\text { no } \\
\text { evidence }\end{array}$ & yes & 0.16 & neg & neg & + & - \\
\hline & 17 & Multiple myeloma & $\begin{array}{l}\text { no } \\
\text { evidence }\end{array}$ & yes & 0.12 & neg & n.a. & + & - \\
\hline & 18 & $\begin{array}{l}\text { Acute myeloid } \\
\text { leukemia }\end{array}$ & $\begin{array}{l}\text { no } \\
\text { evidence }\end{array}$ & no & 0.18 & neg & neg & + & - \\
\hline & 19 & $\begin{array}{l}\text { Acute myeloid } \\
\text { leukemia }\end{array}$ & $\begin{array}{l}\text { no } \\
\text { evidence }\end{array}$ & yes & 0.18 & neg & n.a. & ++ & - \\
\hline
\end{tabular}

* Patients with true negative results $(\mathrm{n}=9)$ not displayed: all 9 had also negative results with new LFD, PCR and culture. 1/9 had a false positive BALF GM result (1.04 ODI). With regard to underlying diseases 4/9 had acute myeloid leukemia, 2/9 had acute lymphoid leukemia, each 1/9 had non-Hodgkin's lymphoma, multiple myeloma, and severe aplastic anemia).

$\S$ Defined according to revised EORTC/MSG criteria (14)

Abbreviations: AIDS = acquired immunodeficiency syndrome; BALF = bronchoalveolar lavage fluid; COPD = chronic obstructive pulmonary disease; GM = galactomannan; IPA - invasive pulmonary aspergillosis; LFD = Aspergillus-specific lateral-flow device test; n.a. = not available; neg = negative; ODI = optical density index; pos = positive .

This article is protected by copyright. All rights reserved. 
FIGURE 1 Results with the new Aspergillus-specific lateral-flow device test ranging (from the left to the right) from negative (-) to weak positive $(+)$, moderate positive $(++)$ and strong positive $(+++)+$

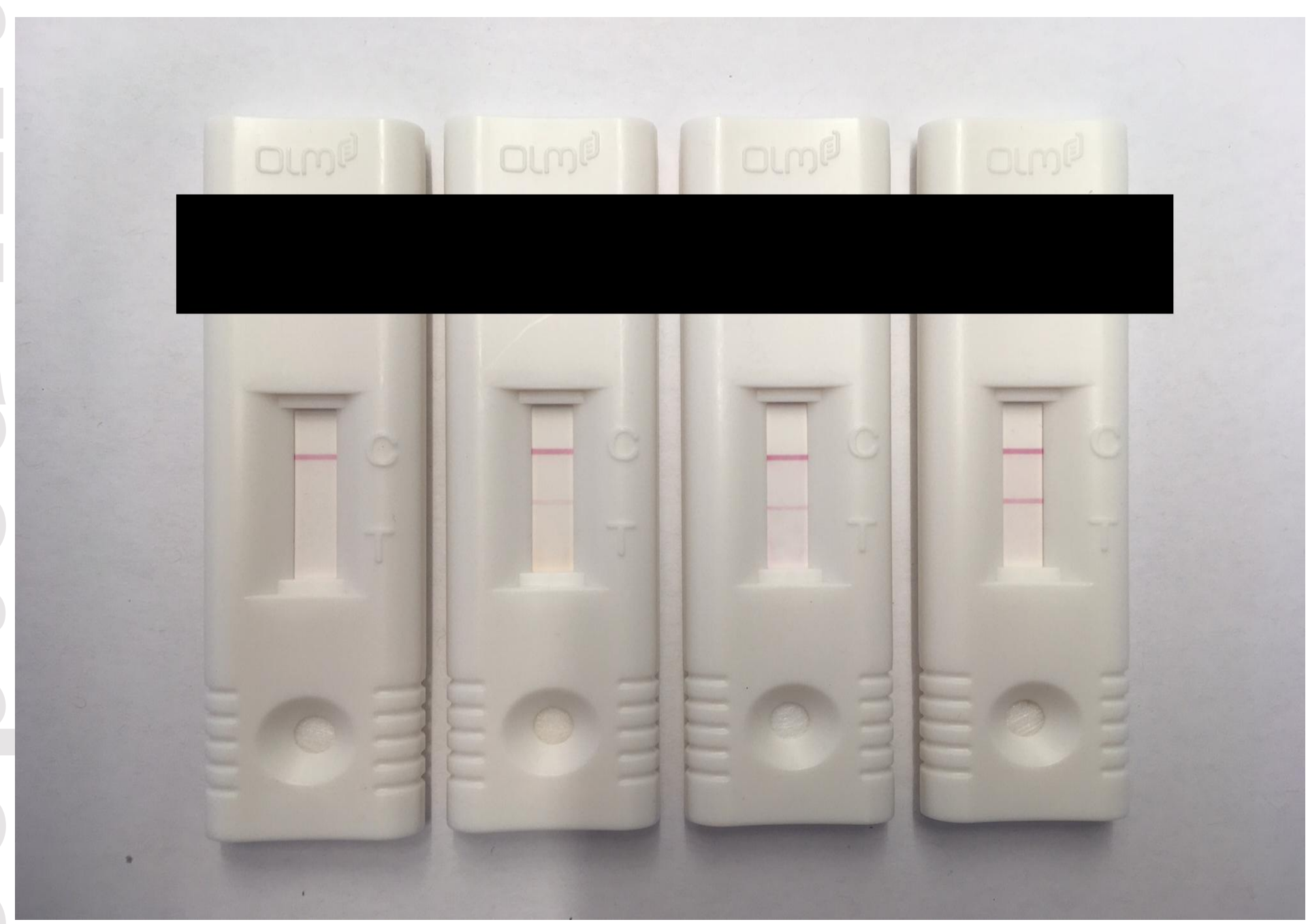

This article is protected by copyright. All rights reserved. 